\title{
Front Matter: Volume 8357
}

, "Front Matter: Volume 8357," Proc. SPIE 8357, Detection and Sensing of Mines, Explosive Objects, and Obscured Targets XVII, 835701 (22 June 2012); doi: $10.1117 / 12.934820$

Event: SPIE Defense, Security, and Sensing, 2012, Baltimore, Maryland, United States 


\title{
PROCEEDINGS OF SPIE
}

\section{Defection and Sensing of Mines, Explosive Objects, and Obscured Targets XVII}

\author{
J. Thomas Broach \\ John H. Holloway, Jr. \\ Editors
}

23-27 April 2012

Baltimore, Maryland, United States

Sponsored and Published by

SPIE 
The papers included in this volume were part of the technical conference cited on the cover and title page. Papers were selected and subject to review by the editors and conference program committee. Some conference presentations may not be available for publication. The papers published in these proceedings reflect the work and thoughts of the authors and are published herein as submitted. The publisher is not responsible for the validity of the information or for any outcomes resulting from reliance thereon.

Please use the following format to cite material from this book:

Author(s), "Title of Paper," in Detection and Sensing of Mines, Explosive Objects, and Obscured Targets XVII, edited by J. Thomas Broach, John H. Holloway, Jr., Proceedings of SPIE Vol. 8357 (SPIE, Bellingham, WA, 2012) Article CID Number.

ISSN 0277-786X

ISBN 9780819490353

Published by

SPIE

P.O. Box 10, Bellingham, Washington 98227-0010 USA

Telephone +1 3606763290 (Pacific Time) · Fax +1 3606471445

SPIE.org

Copyright (C) 2012, Society of Photo-Optical Instrumentation Engineers

Copying of material in this book for internal or personal use, or for the internal or personal use of specific clients, beyond the fair use provisions granted by the U.S. Copyright Law is authorized by SPIE subject to payment of copying fees. The Transactional Reporting Service base fee for this volume is $\$ 18.00$ per article (or portion thereof), which should be paid directly to the Copyright Clearance Center (CCC), 222 Rosewood Drive, Danvers, MA 01923. Payment may also be made electronically through CCC Online at copyright.com. Other copying for republication, resale, advertising or promotion, or any form of systematic or multiple reproduction of any material in this book is prohibited except with permission in writing from the publisher. The CCC fee code is 0277-786X/12/\$18.00.

Printed in the United States of America.

Publication of record for individual papers is online in the SPIE Digital Library.

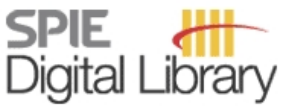

SPIEDigitalLibrary.org

Paper Numbering: Proceedings of SPIE follow an e-First publication model, with papers published first online and then in print and on CD-ROM. Papers are published as they are submitted and meet publication criteria. A unique, consistent, permanent citation identifier (CID) number is assigned to each article at the time of the first publication. Utilization of CIDs allows articles to be fully citable as soon as they are published online, and connects the same identifier to all online, print, and electronic versions of the publication. SPIE uses a six-digit CID article numbering system in which:

- The first four digits correspond to the SPIE volume number.

- The last two digits indicate publication order within the volume using a Base 36 numbering system employing both numerals and letters. These two-number sets start with 00, 01, 02, 03, 04, $05,06,07,08,09,0 A, 0 B \ldots 0 Z$, followed by 10-1Z, 20-2Z, etc.

The CID number appears on each page of the manuscript. The complete citation is used on the first page, and an abbreviated version on subsequent pages. Numbers in the index correspond to the last two digits of the six-digit CID number. 


\section{Contents}

\section{SESSION 1 ELECTROMAGNETIC INDUCTION I}

835702 Pedemis: a portable electromagnetic induction sensor with integrated positioning [8357-01] B. E. Barrowes, U.S. Army Corps of Engineers (United States) and Thayer School of Engineering at Dartmouth College (United States); F. Shubitidze, Thayer School of Engineering at Dartmouth College (United States); T. M. Grzegorczyk, Delpsi, LLC (United States); P. Fernández, Thayer School of Engineering at Dartmouth College (United States); K. O'Neill, U.S. Army Corps of Engineers (United States) and Thayer School of Engineering at Dartmouth College (United States)

835703 Optimizing EMI transmitter and receiver configurations to enhance detection and identification of small and deep metallic targets [8357-02] J. P. Fernández, Consultant (United States); B. Barrowes, Thayer School of Engineering at Dartmouth (United States) and U.S. Army Corps of Engineers (United States); A. Bijamov, Thayer School of Engineering at Dartmouth (United States); K. O'Neill, Thayer School of Engineering at Dartmouth (United States) and U.S. Army Corps of Engineers (United States); I. Shamatava, Thayer School of Engineering at Dartmouth (United States) and Sky Research, Inc. (United States); D. A. Steinhurst, Nova Research, Inc. (United States); F. Shubitidze, Thayer School of Engineering at Dartmouth (United States) and Sky Research, Inc. (United States)

835704 Inversion and classification studies of live-site production-level MetalMapper data sets [8357-03]

F. Shubitidze, Sky Research, Inc. (United States) and Thayer School of Engineering at Dartmouth (United States); J. P. Fernández, Thayer School of Engineering at Dartmouth (United States); J. Miller, J. Keranen, Sky Research, Inc. (United States); B. E. Barrowes, Thayer School of Engineering at Dartmouth (United States) and U.S. Army Corps of Engineers (United States); A. Bijamov, Thayer School of Engineering at Dartmouth (United States)

835705 Inversion-free discrimination of unexploded ordnance in real time [8357-04]

F. Shubitidze, Thayer School of Engineering at Dartmouth (United States) and Sky Research, Inc. (United States); J. P. Fernández, Thayer School of Engineering at Dartmouth (United States); I. Shamatava, Thayer School of Engineering at Dartmouth (United States) and Sky Research, Inc. (United States); A. Luperon, Thayer School of Engineering at Dartmouth (United States); B. E. Barrowes, Thayer School of Engineering at Dartmouth (United States) and U.S. Army Corps of Engineers (United States); K. O'Neill, Thayer School of Engineering at Dartmouth (United States) and U.S. Army Corps of Engineers (United States); A. Bijamov, Thayer School of Engineering at Dartmouth (United States) 
835706 Camp Beale live-site handheld-sensor data inversion and classification using advanced EMI models [8357-05]

I. Shamatava, Sky Research, Inc. (United States) and Thayer School of Engineering at Darmouth College (United States); J. P. Fernández, Thayer School of Engineering at Darmouth College (United States); B. E. Barrowes, K. O'Neill Thayer School of Engineering at Darmouth College (United States) and U.S. Army Corps of Engineers (United States); A. Bijamov, Thayer School of Engineering at Dartmouth (United States); F. Shubitidze, Sky Research, Inc. (United States) and Thayer School of Engineering at Dartmouth (United States)

\section{SESSION 2 ELECTROMAGNETIC INDUCTION II}

835707 Large loop EMI sensor for detection of deeply buried munitions in magnetic soils [8357-06] J. S. Miller, Sky Research, Inc. (United States); S. Billings, Sky Research, Inc. (Australia); J. Keranen, G. Schultz, C. Bassani, Sky Research, Inc. (United States)

835708 Feature extraction and processing of spatial frequency-domain electromagnetic induction sensor data for improved landmine discrimination [8357-07]

S. L. Tantum, K. A. Colwell, K. D. Morton, Jr., Duke Univ. (United States); W. R. Scott, Jr., Georgia Institute of Technology (United States); L. M. Collins, P. A. Torrione, Duke Univ. (United States)

835709 On the estimation of target depth using the single transmit multiple receive metal detector array [8357-08]

K. C. Ho, Univ. of Missouri-Columbia (United States); P. D. Gader, Univ. of Florida (United States)

8357 OA Robust estimation of the discrete spectrum of relaxations from multiple-electromagnetic induction responses [8357-09]

M.-H. Wei, W. R. Scott, Jr., J. H. McClellan, Georgia Institute of Technology (United States)

$8357 \mathrm{OB}$ Landmine detection using two-tapped joint orthogonal matching pursuits [8357-83]

S. Goldberg, T. Glenn, J. N. Wilson, P. D. Gader, Univ. of Florida (United States)

\section{SESSION 3 ELECTROMAGNETIC INDUCTION III}

8357 OC Progress on a system for measuring wide-band electromagnetic induction responses [8357-10]

W. R. Scott, Jr., M. McFadden, Georgia Institute of Technology (United States)

8357 OD Location and orientation estimation of buried targets using electromagnetic induction sensors [8357-11]

K. Krueger, W. R. Scott, Jr., J. H. McClellan, Georgia Institute of Technology (United States)

8357 OE Induction detection of concealed bulk banknotes [8357-12]

C. Fuller, A. Chen, Univ. of Washington (United States)

8357 OF Pinpointing error analysis of metal detectors under field conditions [8357-13]

K. Takahashi, Tohoku Univ. (Japan); H. Preetz, Leibniz Institute for Applied Geophysics (Germany) 
$8357 \mathrm{OH} \quad$ Experimental investigation of buried landmine detection using time division multiplexing of multibeam laser Doppler vibrometer channels [8357-15]

R. Burgett, V. Aranchuk, I. Aranchuk, The Univ. of Mississippi (United States)

8357 Ol Synthetic aperture acoustic imaging of non-metallic cords [8357-16]

A. A. J. Glean, C. E. Good, J. F. Vignola, J. A. Judge, T. J. Ryan, The Catholic Univ. of America (United States); S. S. Bishop, P. M. Gugino, U.S. Army Night Vision \& Electronic Sensors

Directorate (United States); M. Soumekh, Soumekh Consulting (United States)

SESSION 5 INFRARED AND ELECTRO-OPTIC I

8357 OJ Overview of computational testbed for evaluating electro-optical/infrared sensor systems [8357-17]

R. V. Kala, J. R. Fairley, S. J. Price, J. R. Ballard, Jr., A. R. Carrillo, S. E. Howington, O. J. Eslinger, A. M. Hines, R. A. Goodson, U.S. Army Corps of Engineers (United States)

8357 OK Examining the sensitivity of simulated surface temperatures due to meteorological conditions [8357-18]

O. J. Eslinger, C. Winton, A. M. Hines, R. Goodson, S. E. Howington, R. Kala, J. Fairley, S. Price, U.S. Army Corps of Engineers (United States); K. Elder, U.S. Forest Service (United States)

$8357 \mathrm{OL} \quad$ Cloud cover effects on physical soil temperatures with buried targets [8357-19]

Z. Derzko, O. Nguyen, C. Phan, R. Lydic, J. T. Broach, T. Moore, U.S. Army Night Vision \& Electronic Sensors Directorate (United States)

8357 OM Rain effects on physical soil temperatures with buried targets [8357-20]

Z. Derzko, O. Nguyen, C. Phan, R. Lydic, J. T. Broach, U.S. Army Night Vision \& Electronic Sensors Directorate (United States)

$8357 \mathrm{ON} \quad$ Schedule optimization for IR detection of buried targets [8357-21]

Z. Derzko, U.S. Army Night Vision \& Electronic Sensors Directorate (United States);

J. B. Eylander, U.S. Army Corps of Engineers (United States); J. T. Broach, U.S. Army Night

Vision \& Electronic Sensors Directorate (United States)

SESSION 6 INFRARED AND ELECTRO-OPTIC II

835700 The evaluation of hyperspectral imaging for the detection of person-borne threat objects over the 400nm to $1700 \mathrm{~nm}$ spectral region [8357-22]

C. C. Cooksey, J. E. Neira, D. W. Allen, National Institute of Standards and Technology (United States)

8357 OP Role of moisture and density of sand for microwave enhancement of thermal detection of buried mines [8357-23]

W. Swiderski, P. Hlosta, J. Jarzemski, L. Szugajew, Military Institute of Armament Technology (Poland); J. Usowicz, Nicolaus Copernicus Univ. (Poland) 
$8357 \mathrm{OQ} \quad$ Buried mine detection using fractal geometry analysis to the LWIR successive line scan data image [8357-24]

K. Araki, Ministry of Defence (Japan)

SESSION 7 INFRARED AND ELECTRO-OPTIC III

8357 OR Three-dimensional material identification and hazard detection with shortwave infrared supercontinuum-based spectral ladar [8357-25]

M. A. Powers, General Dynamics (United States)

8357 OS Road detection and buried object detection in elevated EO/IR imagery [8357-26]

L. Kennedy, M. P. Kolba, J. R. Walters, Signal Innovations Group, Inc. (United States)

8357 OT Anomaly detection ensemble fusion for buried explosive material detection in forward looking infrared imaging for addressing diurnal temperature variation [8357-27]

D. T. Anderson, Mississippi State Univ. (United States); K. Stone, J. M. Keller, Univ. of Missouri-Columbia (United States); J. Rose, Mississippi State Univ. (United States)

\section{SESSION 8 BULK EXPLOSIVE DETECTION}

8357 OV Improved thermal neutron activation sensor for detection of bulk explosives [8357-29]

J. E. McFee, A. A. Faust, Defence R\&D Canada, Suffield (Canada); H. R. Andrews,

E. T. H. Clifford, Bubble Technology Industries Inc. (Canada); C. M. Mosquera, Defence R\&D Canada, Suffield (Canada)

8357 OW Feasibility of culvert IED detection using thermal neutron activation [8357-30]

A. A. Faust, J. E. McFee, Defence Research and Development Canada, Suffield (Canada);

E. T. H. Clifford, H. R. Andrews, Bubble Technology Industries Inc. (Canada); C. M. Mosquera, W. C. Roberts, Defence Research and Development Canada, Suffield (Canada)

8357 OX Pixelated diffraction signatures for explosive detection [8357-31]

D. O'Flynn, C. Reid, C. Christodoulou, Univ. College London (United Kingdom); M. D. Wilson, M. C. Veale, P. Seller, Rutherford Appleton Lab. (United Kingdom); R. Speller, Univ. College London (United Kingdom)

8357 OY Pulse sequences for the detection of RDX at 5.192 MHz: steady state free precession (SSFP) versus free induction decay [8357-32]

T. Schunck, K. Darée, D. Krüger, R. Himmelsbach, L. Merlat, Institut Franco-Allemand de Recherches de Saint-Louis (France)

$8357 \mathrm{OZ} \quad$ Novel approaches in nuclear magnetic/quadrupole resonance techniques for explosives detection [8357-33]

B. Z. Rameev, Gebze Institute of Technology (Turkey) and E.K. Zavoisky Physical-Technical Institute (Russian Federation); G. V. Mozzhukhin, Gebze Institute of Technology (Turkey) and Kazan Power State Engineering Univ. (Russian Federation); R. R. Khusnutdinov, Gebeze Institute of Technology (Turkey) and E.K. Zavoisky Physical-Technical Institute (Russia) and Kazan Power State Engineering Univ. (Russian Federation); B. Aktas, Gebze Institute of Technology (Turkey); A. B. Konov, D. D. Gabidullin, N. A. Krylatyh, Y. V. Fattakhov, K. M. Salikhov, E.K. Zavoisky Physical-Technical Institute (Russian Federation) 
835710 Landmine detection by 3D GPR system [8357-34]

M. Sato, Y. Yokota, K. Takahashi, Tohoku Univ. (Japan); M. Grasmueck, Univ. of Miami (United States)

$835712 \quad$ Modeling GPR data from lidar soil surface profile [8357-36]

B. Burns, W. W. Clark, I. McMichael, U.S. Army Night Vision \& Electronic Sensors Directorate (United States)

835713 Forward looking GPR sidelobe reduction using L1-norm minimization [8357-37]

B. Burns, U.S. Army Night Vision \& Electronic Sensors Directorate (United States)

835714 A novel forward and backward scattering wave measurement system for optimizing GPR standoff mine/IED detector [8357-38]

Y. Fuse, Ministry of Defence (Japan)

SESSION 10 A MELANGE OF INTERESTING TECHNIQUES II

835716 Simulation study of $\mathrm{x}$-ray backscatter imaging of pressure-plate improvised explosive devices [8357-40]

J. van den Heuvel, F. Fiore, NATO C3 Agency (Netherlands)

835718 Laser neutralization of surface and buried munitions [8357-42]

J. D. Habersat, B. W. Schilling, J. Alexander, U.S. Army Night Vision \& Electronic Sensors

Directorate (United States); T. Lehecka, The Pennsylvania State Univ. Electro-Optics Ctr. (United States)

\section{SESSION 11 HAND-HELD SYSTEMS}

8357 1A ALIS deployment in Cambodia [8357-45]

M. Sato, K. Takahashi, Tohoku Univ. (Japan)

8357 IB Investigation of the effects of operator technique on handheld sensor data for landmine detection [8357-46]

S. L. Tantum, K. D. Morton, Jr., L. M. Collins, P. A. Torrione, Duke Univ. (United States)

\section{SESSION 12 STAND-OFF DETECTION TECHNOLOGIES I}

8357 1C Evaluation and improvement of spectral features for the detection of buried explosive hazards using forward-looking ground-penetrating radar [8357-48]

J. Farrell, T. C. Havens, K. C. Ho, J. M. Keller, Univ. of Missouri-Columbia (United States);

T. T. Ton, D. C. Wong, U.S. Army Night Vision \& Electronic Sensors Directorate (United States);

M. Soumekh, Univ. of New York at Buffalo (United States) 
8357 ID Multiple kernel learning for explosive hazard detection in forward-looking ground-penetrating radar [8357-49]

T. C. Havens, K. Stone, D. T. Anderson, J. M. Keller, K. C. Ho, Univ. of Missouri-Columbia (United States); T. T. Ton, D. C. Wong, U.S. Army Night Vision \& Electronic Sensors Directorate (United States); M. Soumekh, Univ. of New York at Buffalo (United States)

$83571 \mathrm{E}$ An automatic detection system for buried explosive hazards in FL-LWIR and FL-GPR data [8357-50]

K. Stone, J. M. Keller, Univ. of Missouri-Columbia (United States); D. T. Anderson, Mississippi State Univ. (United States); D. B. Barclay, Univ. of Missouri-Columbia (United States)

8357 IF Fusion of UHF-SAR with lidar elevation for precise buried object detection [8357-51] A. K. Shaw, Wright State Univ. (United States) and Gitam Technologies, Inc. (United States); D. Rahn, Gitam Technologies, Inc. (United States); R. Depoy, Wright State Univ. (United States)

8357 1G Optimizing a lab-on-a-fiber optic device for trace TNT explosive detection [8357-52] J. Ma, A. Kos, W. J. Bock, Univ. du Québec en Outaouais (Canada); W. Hao, Z. Y. Wang, Carleton Univ. (Canada)

8357 1H Detection limit of imaging Raman spectroscopy [8357-53] M. Nordberg, Swedish Defence Research Agency (Sweden) and Royal Institute of Technology (Sweden); E. Ceco, S. Wallin, H. Östmark, Swedish Defence Research Agency (Sweden)

835711 Time-of-flight mass spectrometry for explosives trace detection [8357-54]

A. Pettersson, A. Elfving, M. Elfsberg, T. Hurtig, N. Johansson, A. Al-Khalili, P. Käck, S. Wallin, H. Östmark, Swedish Defence Research Agency (Sweden)

8357 1 J Quantum dot material for the detection of explosive-related chemicals [8357-55] V. P. Schnee, M. D. Woodka, D. Pinkham, U.S. Army Night Vision \& Electronic Sensors Directorate (United States)

8357 1K Feature optimization in chemometric algorithms for explosives detection [8357-57] D. W. Pinkham, J. R. Bonick, M. D. Woodka, U.S. Army Night Vision \& Electronic Sensors Directorate (United States)

\section{SESSION 14 STAND-OFF DETECTION TECHNOLOGIES II}

8357 IL Line matching for automatic change detection algorithm [8357-58]

J. Dhollande, D. Monnin, L. Gond, Institut Franco-Allemand de Recherches de Saint-Louis (France); C. Cudel, S. Kohler, A. Dieterlen, Univ. de Haute Alsace (France)

$83571 \mathrm{M}$ Change-based threat detection in urban environments with a forward-looking camera [8357-59]

K. Morton, Jr., C. Ratto, J. Malof, M. Gunter, L. Collins, P. Torrione, Duke Univ. (United States) 
8357 iN Optimized feature-detection for on-board vision-based surveillance [8357-60]

L. Gond, D. Monnin, A. Schneider, Institut Franco-Allemand de Recherches de Saint-Louis (France)

835710 Processing forward-looking data for anomaly detection: single-look, multi-look, and spatial classification [8357-61]

J. M. Malof, K. D. Morton, Jr., L. M. Collins, P. A. Torrione, Duke Univ. (United States)

\section{SESSION 15 MARINE ENVIRONMENT}

8357 IP Inspection of the objects on the sea floor for the presence of explosives [8357-62]

V. Valkovic, A.C.T.d.o.o (Croatia); D. Sudac, Institute Ruder Boškovic (Croatia); R. Kollar, A.C.T.d.o.o (Croatia); Z. Domitran, Univ. of Zagreb (Croatia); K. Nad, J. Obhodas, Institute Ruder Boškovic (Croatia)

$83571 Q$ Detection of floating mines in infrared sequences by multiscale geometric filtering [8357-63]

D. Florins, A. Manzanera, Ecole Nationale Supérieure de Techniques Avancées (France)

8357 IR Correction of underwater pincushion distortion by a compensating camera lens [8357-64] H. R. Suiter, Naval Surface Warfare Ctr. Panama City Div. (United States)

8357 is Future planning and evaluation for automated adaptive minehunting: a roadmap for mine countermeasures theory modernization [8357-65]

G. A. Garcia, Naval Surface Warfare Ctr. Panama City Div. (United States); T. A. Wettergren, Naval Undersea Warfare Ctr. Newport Div. (United States)

8357 1T Sensor array and preconcentrator for the detection of explosives in water [8357-66] M. D. Woodka, J. C. Shpil, V. P. Schnee, U.S. Army Night Vision \& Electronic Sensors Directorate (United States); J. M. P. Polcha, Corbin Co. (United States)

8357 IU Integration of lidar with the NIITEK GPR for improved performance on rough terrain [8357-67] C. R. Ratto, K. D. Morton, Jr., Duke Univ. (United States); I. T. McMichael, B. P. Burns, W. W. Clark, U.S. Army Night Vision \& Electronic Sensors Directorate (United States); L. M. Collins, P. A. Torrione, Duke Univ. (United States)

8357 IV Ground tracking using Microsoft Kinect sensor for ground-penetrating radar [8357-68] R. H. Luke, M. A. Cumo, U.S. Army Night Vision \& Electronic Sensors Directorate (United States)

8357 IW Extracting edge histogram detector features from ground penetrating radar data without ground alignment [8357-69]

J. Wood, J. Wilson, J. Bolton, Univ. of Florida (United States)

8357 IX Efficient multiple layer boundary detection in ground penetrating radar data using an extended Viterbi algorithm [8357-70]

B. Smock, J. Wilson, Univ. of Florida (United States) 
8357 IY Image registration and change detection feasibility study with ground penetrating radar [8357-71]

R. Mueller, S. Lauziere, M. Khanin, NIITEK, Inc. (United States); M. Hibbard, CoVar Applied Technologies, Inc. (United States)

\section{SESSION 17 SIGNAL PROCESSING II}

$83571 \mathrm{Z}$ Keypoint-based image processing for landmine detection in GPR data [8357-72]

R. T. Sakaguchi, K. D. Morton, Jr., L. M. Collins, P. A. Torrione, Duke Univ. (United States)

835720 Weighted principal component analysis for real-time background removal in GPR data [8357-73]

Y. P. Shkolnikov, Exponent, Inc. (United States)

835721 Multiple instance learning for landmine detection using ground penetrating radar [8357-74] A. Manandhar, K. D. Morton, Jr., L. M. Collins, P. A. Torrione, Duke Univ. (United States)

835722 Incorporation of operator knowledge for improved HMDS GPR classification [8357-75]

L. Kennedy, J. R. McClelland, J. R. Walters, Signal Innovations Group (United States)

835723 A Bayesian method for discriminative context-dependent fusion of GPR-based detection algorithms [8357-76]

C. R. Ratto, K. D. Morton, Jr., L. M. Collins, P. A. Torrione, Duke Univ. (United States)

\section{SESSION 18 SIGNAL PROCESSING III}

835726 Classification by using Prony's method with a polynomial model [8357-80]

R. Mueller, W. Lee, J. Okamitsu, NIITEK Inc. (United States)

835727 QSCAN: method for real-time anomaly detection using GPR imaging (Quick-Scan) [8357-81]

A. Etebari, NIITEK, Inc. (United States)

835728 Evaluation of various feature extraction methods for landmine detection using hidden Markov models [8357-82]

A. Hamdi, H. Frigui, Univ. of Louisville (United States)

\section{POSTER SESSION}

835729 Improving detection range via correlation of long PN codes [8357-84]

S. Subedi, Z. Wang, Y. R. Zheng, Missouri Univ. of Science and Technology (United States)

Author Index 


\title{
Conference Committee
}

\author{
Symposium Chair
}

Kevin P. Meiners, Office of the Secretary of Defense (United States)

Symposium Cochair

Kenneth R. Israel, Lockheed Martin Corporation (United States)

Conference Chairs

J. Thomas Broach, U.S. Army Night Vision \& Electronic Sensors Directorate (United States)

John H. Holloway, Jr., Naval Surface Warfare Center Panama City Division (United States)

Program Committee

Benjamin Barrowes, U.S. Army Corps of Engineers (United States)

Steven S. Bishop, U.S. Army Night Vision \& Electronic Sensors Directorate (United States)

Leslie M. Collins, Duke University (United States)

Gerald J. Dobeck, Naval Surface Warfare Center Panama City Division (United States)

Paul Gader, University of Florida (United States)

Jan M. H. Hendrickx, New Mexico Institute of Mining and Technology (United States)

James M. Keller, University of Missouri-Columbia (United States)

Aaron LaPointe, U.S. Army Night Vision \& Electronic Sensors Directorate (United States)

John E. McFee, Defence Research and Development Canada (Canada)

Henric Östmark, Swedish Defence Research Agency (Sweden)

Motoyuki Sato, Tohoku University (Japan)

Mehmet Sezgin, TÜBITAK Marmara Research Center (Turkey)

Waymond R. Scott, Jr., Georgia Institute of Technology (United States)

Harold R. Suiter, Naval Surface Warfare Center Panama City Division (United States)

Richard C. Weaver, U.S. Army Night Vision \& Electronic Sensors

Directorate (United States) 
Session Chairs

1 Electromagnetic Induction I

Benjamin Barrowes, U.S. Army Corps of Engineers (United States)

Jon Miller, Sky Research, Inc. (United States)

2 Electromagnetic Induction II

Benjamin Barrowes, U.S. Army Corps of Engineers (United States)

Jon Miller, Sky Research, Inc. (United States)

3 Electromagnetic Induction III

Waymond R. Scott, Jr., Georgia Institute of Technology (United States)

Benjamin Barrowes, U.S. Army Corps of Engineers (United States)

$4 \quad$ A Melange of Interesting Techniques I

Steven S. Bishop, U.S. Army Night Vision \& Electronic Sensors Directorate (United States)

5 Infrared and Electro-Optic I

Owen J. Eslinger, U.S. Army Engineer Research and Development

Center (United States)

J. Thomas Broach, U.S. Army Night Vision \& Electronic Sensors

Directorate (United States)

6 Infrared and Electro-Optic II

Neal E. Blackwell, U.S. Army Night Vision \& Electronic Sensors

Directorate (United States)

$7 \quad$ Infrared and Electro-Optic III

Neal E. Blackwell, U.S. Army Night Vision \& Electronic Sensors

Directorate (United States)

8 Bulk Explosive Detection

John E. McFee, Defence Research and Development Canada, Suffield (Canada)

9 Radar

Brian P. Burns, U.S. Army Night Vision \& Electronic Sensors Directorate (United States)

Brian C. Barlow, U.S. Army Night Vision \& Electronic Sensors Directorate (United States)

10 A Melange of Interesting Techniques II

Steven S. Bishop, U.S. Army Night Vision \& Electronic Sensors Directorate (United States) 
11 Hand-Held Systems

Charles A. Amazeen, U.S. Army Night Vision \& Electronic Sensors

Directorate (United States)

12 Stand-Off Detection Technologies I

James M. Keller, University of Missouri-Columbia (United States)

13 Trace Particle/Vapor Explosive Sensing

Aaron LaPointe, U.S. Army Night Vision \& Electronic Sensors Directorate (United States)

Anna K. Pettersson, Swedish Defence Research Agency (Sweden)

14 Stand-Off Detection Technologies II

Robert H. Luke III, U.S. Army Night Vision \& Electronic Sensors

Directorate (United States)

15 Marine Environment

Harold R. Suiter, Naval Surface Warfare Center Panama City Division (United States)

Gerald J. Dobeck, Naval Surface Warfare Center Panama City Division (United States)

16 Signal Processing I: GPR Ground Tracking and Change Detection

Richard C. Weaver, U.S. Army Night Vision \& Electronic Sensors

Directorate (United States)

17 Signal Processing II

Robert H. Luke III, U.S. Army Night Vision \& Electronic Sensors

Directorate (United States)

Peter A. Torrione, Duke University (United States)

18 Signal Processing III

Pete Howard, U.S. Army Night Vision \& Electronic Sensors Directorate (United States)

Christopher R. Ratto, Duke University (United States) 
Proc. of SPIE Vol. $8357835701-14$

Downloaded From: https://www.spiedigitallibrary.org/conference-proceedings-of-spie on 26 Apr 2023 Terms of Use: https://www.spiedigitallibrary.org/terms-of-use 